\title{
Continuity of care and its determinants of routine maternal and newborn health visits in Nepal: Evidence from a nationally representative household survey
}

\section{Resham Bahadur Khatri ( $\nabla$ rkchettri@gmail.com )}

Health Social Science and Development Research Institute, Kathmandu

\section{Rajendra Karkee}

School of Public Health and Community Medicine, BP Koirala Institute of Health Sciences, Dharan, Jo Durham

School of Public Health and Social Work, Queensland University of Technology, Brisbane

\section{Yibeltal Assefa}

School of Public Health, Faculty of Medicine, University of Queensland, Brisbane

\section{Research Article}

Keywords: continuum of care, maternal and newborn health, determinants, routine visits, essential interventions

Posted Date: June 4th, 2021

DOI: https://doi.org/10.21203/rs.3.rs-272766/v1

License: (c) (1) This work is licensed under a Creative Commons Attribution 4.0 International License. Read Full License 
1 Continuity of care and its determinants of routine maternal and newborn

2 health visits in Nepal: Evidence from a nationally representative household

3 survey

4

5 Resham B Khatri ${ }^{1,2 *}$, Rajendra Karkee ${ }^{3}$, Jo Durham ${ }^{1,4}$, Yibeltal Assefa ${ }^{1}$

6

7 ' ${ }^{1}$ School of Public Health, Faculty of Medicine, University of Queensland, Brisbane, 8 Australia.

$9 \quad{ }^{2}$ Health Social Science and Development Research Institute, Kathmandu, Nepal

${ }^{3}$ School of Public Health and Community Medicine, BP Koirala Institute of Health

11 Sciences, Dharan, Nepal.

$12{ }^{4}$ School of Public Health and Social Work, Queensland University of Technology,

13 Brisbane, Australia

14

*Corresponding author (E-mail): rkchettri@gmail.com

Word Count: 5803 (excluding title page, abstract, figure, references, and supplementary file)

DISCLAIMER: Views presented in this article are solely those of the authors and do not represent the views, interests, or funded work of the organisations where authors 22 affiliated. 


\section{Abstract}

\section{Background}

3 Maternal and newborn health $(\mathrm{MNH})$ is a priority health issue in Nepal, has high maternal and neonatal deaths. Maternal and neonatal deaths can be prevented through uptake of essential antenatal, intrapartum, and postnatal interventions received during routine $\mathrm{MNH}$ visits. Not all women, however, receive all recommended routine visits across the $\mathrm{MNH}$ Continuum of Care $(\mathrm{CoC})$ in Nepal.

8 This study examined the patterns and determinants of (dis)continuity of care across 9 the MNH continuum.

\section{Methods}

11 The study included 1,978 women aged 15-49 years who had a live birth in the two years preceding the survey. Data were derived from the Nepal Demographic and Health Survey (NDHS) 2016. The outcome variable was (dis)continuity of care at different stages of MNH visits (at least four antenatal care (4ANC) visits, institutional delivery, and postnatal care (PNC) visit). Several structural, intermediary and health system explanatory variables were included in the analysis. Multinomial logistic regression analysis was conducted, and the magnitude of (dis)continuity of care was reported as relative risk ratios (RR) with 95\% confidence intervals (Cls). The statistical significance level was set $p<0.05$.

\section{Results}

21 More than two-in-five (41\%) women in Nepal received all three MNH visits across the CoC. There was high risk of discontinuity of care during months or weeks prior to childbirth or around childbirth. Higher risk of discontinuation across the CoC was reported among women of disadvantaged ethnic groups, lower wealth status and illiterate. Similarly, women who speak Bhojpuri, provinces six and seven, who had higher birth order $(\geq 4)$, who involved in agricultural sector, had unwanted last birth had higher risk of discontinuation of MNH visits. Women did not complete all MNH visits if they had poor awareness on health mother groups and if they perceived problem of not having female healthcare providers.

\section{Conclusions}

Women had poor completion of all routine MNH visits. High discontinuation was observed among disadvantaged groups across the COC. Regular monitoring using the composite indicator of continuity of care through routine health management

34 information system is required. Program approaches should focus on disadvantaged 
1 women to improve the completion of routine $\mathrm{MNH}$ visits and uptake of essential 2 interventions.

3 Keywords: continuum of care, maternal and newborn health, determinants, routine

4 visits, essential interventions

\section{Introduction}

6 Maternal and newborn health $(\mathrm{MNH})$ is a priority public health issue in low- and

7 lower-middle-income countries (LMICs). Most maternal and newborn deaths occur in

8 Sub-Saharan Africa and South Asia [1]. The majority of the maternal and newborn

9 deaths could be prevented through uptake of essential antenatal, intrapartum and postnatal interventions [2]. The World Health Organization (WHO) recommends women should receive health interventions during routine $\mathrm{MNH}$ visits such as at least four antenatal care (4ANC) visits, institutional delivery assisted by skilled health providers [3], at least three PNC visits within the first week after childbirth [4].

A study of 75 LMICs high burden of maternal and neonatal deaths estimated increased access and quality of essential $\mathrm{MNH}$ interventions could reduce up to $71 \%$ of neonatal deaths, $33 \%$ of stillbirths, and $54 \%$ of maternal deaths annually [5]. The coverage of routine $\mathrm{MNH}$ visits during maternity period, however, is often low and characterised by high rates of discontinuation along the continuum of care (CoC). For example, the completion of all routine MNH visits was low in several LMICs [69], including in Ghana [10], Cambodia [6], and Tanzania [11]. In Tanzania, 90\% dropout was reported from first ANC visit to PNC visit; while the highest (55\%) proportion was seen from institutional delivery to a PNC visit [11].

Maternal and newborn health is continuum of care (CoC) from the life cycle perspective [8]. This perspective of $\mathrm{CoC}$ describes delivery of health services from conception through to birth, and childhood- adolescence-adulthood period. The antenatal, intrapartum, and postnatal is a shorter version of CoC. This period is vital for health status of mothers and newborns, and is the combined construct from survival and health service delivery [7]. It is arguably a single entity except for their biological differences; for instance, interventions received by pregnant women can affect health newborns. In the MNH continuum, mid-level health workers can provide essential MNH interventions services up to the first level of health system (peripheral health facilities) and community level. The combined coverage of 4 ANC visits, 
1 institutional delivery, and first PNC visit can be considered as the marker of the CoC 2 of $\mathrm{MNH}[12-14]$.

3 Globally, the $\mathrm{CoC}$ in $\mathrm{MNH}$ has received substantial attention in research, policy and 4 programs over the past two decades [7]. For instance, the sustainable development 5 goal three (SDG3) states universal coverage of quality $\mathrm{MNH}$ services across the CoC (target 3.8) $[10,15]$. Out of nine tracer services in SDG3, two are related to 7 MNH services, such as childbirth assisted by skilled health attendants and health 8 facility delivery [16]. Thus, the assessment of composite coverage of all routine visits 9 is essential to track the coverage of tracer MNH services and SDG3 target.

10 11

Nepal has the highest maternal and neonatal death rates within South Asia [17, 18]. Annually 259 (per 100,000 live births) women die due to pregnancy and childbirthrelated issues, and 21 (per 1000 live births) newborn die within the first month of birth in Nepal [19]. High maternal and neonatal deaths in Nepal may be contributed by low coverage of $\mathrm{MNH}$ visits, thereby lack of uptake of essential interventions across MNH continuum. Past evidence in Nepal showed poor access to MNH services and poor completion of MNH visits. For instance, Nepal Demographic and Health Survey (NDHS) 2016 reported 70\% of pregnant women received 4ANC visits, and nearly two in five women received institutional delivery and first PNC visit within 48 hours of childbirth [19]. A study in 2019 reported only $40 \%$ women completed all routine $\mathrm{MNH}$ visits, i.e., 4ANC visits, institutional delivery, and PNC visit [8]. The same study reported among rural women, and those with higher birth order (more than two children) had lower odds of MNH visits across the $\mathrm{CoC}$ [8]. Another study reported women of wealth status and illiterate women low the completion of $\mathrm{MNH}$ visits across the $\mathrm{CoC}[20]$. Other studies revealed that the completion of 4ANC visits contributed to uptake of institutional delivery [21] and PNC visit [22], and women who received institutional delivery services were more likely to receive PNC visit [23-25]. A qualitative exploration of access to PNC observed Nepalese women might only prioritise PNC if they experience complications [26]. However, there is a dearth of evidence on the patterns of (dis)continuity of care at different stages of $\mathrm{MNH}$ visits across the MNH CoC and their associated determinants. Thus, this study examined the patterns of inequity of (dis)continuity of all $\mathrm{MNH}$ visits and their determinants across the $\mathrm{CoC}$ in Nepal. 


\section{Methods}

\section{Data source and sampling design}

3 The Nepal Demographic and Health Survey (NDHS) 2016 data [19] were used in this

4 study. The NDHS is a nationally representative cross-sectional survey conducted by

5 the MOHP (Nepal) in 2016 to identify the health status and services coverage of

6 primary health care programs, especially family planning, reproductive, newborn,

7 child health and nutrition.

8 A more detailed sampling design is described in the NDHS 2016 report [19]. Briefly,

9 the NDHS 2016 identified rural and urban sampling strata from seven provinces. The survey adopted a two-stage sampling design. The first stage involved the systematic selection of 383 clusters (primary sampling units) with probability proportional to size from urban and rural strata (14 strata). The second stage consisted of selecting 30 households per cluster through a systematic sampling from a selected household. Of 11, 203 households selected, 11,040 households participated in the survey. Of 13, 089 women aged 15-49 years identified for the interview, 12,862 responded (response rate of $98 \%$ ). Of them, total 3,998 women had a live birth five years preceding the survey. However, this study restricted to 1,978 women aged 15-49 years who had a live birth in the two years preceding the survey. The NDHS 2016 collected information on pregnancy, childbirth, and postnatal care from women.

\section{Conceptual framework of the study}

Based on the review of previous conceptual frameworks [27-29], a conceptual framework was developed for this study (Figure 1). The conceptual framework comprises inputs, outputs, and outcomes. Inputs include different contexts and mechanisms including several determinants, systems, institutional and individuals level factors that can contribute to health outputs, for instance, may produce outputs of (dis)continuity of care and broadly is categorised in three domains: structural, intermediary and health system. Structural domain covers sociopolitical factors (e.g., governance, wealth status, and ethnicity), usually rooted in the distribution of power and resources. Intermediary domain includes factors affecting conditions of heath (non-health sector factors, e.g., geography, transportation, biological and behavioural factors) that affect individual's daily working and living conditions. Structural factors can intermediate non-health sector and can influence the health system variables. 
1 Health system domain includes variables that affect the provision and delivery of

2 quality health services.

Inputs (contexts, and mechanisms): Determinants, systems, institutions, individuals

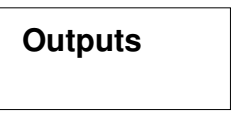

Outcomes

4

5

\section{Structural factors:}

Governance, macroeconomic and social policies, culture and societal values, individuals' characteristics (e.g. social class, gender, education, occupation,

Intermediary factors:

Factors that influence conditions of health, daily lives, residence, province

language, media exposure and awareness on health, individuals' behavioural, biological (e.g., age) and

Figure 1 A conceptual framework adapted and modified from WHO's commission of social determinants of health (WHO 2010).

\section{Study variables}

17 Independent variables included the characteristics of women and their health care experiences (Supplementary file, Table 1). As guided by the conceptual framework, independent variables were grouped into three domains: structural, intermediary, and health system. The structural variables included women's ethnicity, wealth status, education, religion, maternal occupation, perceived violence, decision-making for at least one of three areas (healthcare, purchasing, and movement) and sex of household head. Intermediary variables were women's language, maternal age, residence, provinces, region, birth order, sex of child, access to bank account, media exposure, perceived problem of distance to health facilities, and intended birth of the last child. The health system variables included the women's (perceived) problem of not having female health providers, awareness of health mothers' groups, mode of delivery. 
1 Taking reference from past studies [24, 30-32], we further categorised ethnicity,

2 education, wealth status. The Government of Nepal has categorised 123 ethnicities

3 into six broader categories: i) Dalits (untouchable), ii) disadvantaged indigenous, iii)

4 disadvantaged non-Dalit Terai caste groups, iv) religious minorities (Muslims), v)

5 relatively advantaged indigenous, and vi) upper caste groups. These broader

6 categories of ethnicities were merged into two groups according to their comparative

7 privileges: disadvantaged ethnicities (includes Dalit, Muslims, and Terai caste,

8 disadvantaged Janajatis) and advantaged ethnicities (includes Brahmin/Chhetri,

9 advantaged Janajatis). Maternal education was categorised into illiterate (who cannot read and write), and primary (up to grade eight), and secondary and higher (who have education of grade nine or higher). In the NDHS 2016, wealth quintiles were constructed using principal component analysis (PCA) based on more than 40asset items being owned by households. In this study, these wealth quintiles were merged into two groups, such as the lowest two quintiles as Poor (lower $40 \%$ ), and upper three quintiles as Rich (upper 60\%).

This study had one outcome variable with four mutually exclusive categories: discontinued before completing 4ANC visits=1; completed 4ANC visits but discontinued before completing institutional delivery $=2$; completed $4 \mathrm{ANC}$ visits and institutional delivery but discontinued before completing PNC visit=3; completed all three $\mathrm{MNH}$ visits=0 (reference category).

\section{Statistical analysis}

Multinomial logistic regression analysis was conducted, and the magnitude of (dis)continuity of care was reported as relative risk ratios (RR) with $95 \%$ confidence intervals (Cls). In the analysis, sampling weights (available in the NDHS 2016 dataset) have been calculated and applied, so results are representative at the national as well as strata levels. All analyses were weighted to adjust for the twostaged cluster sampling used in the NDHS 2016 survey (primary sampling unit=383; stratification (strata $=14$; province seven with rural and urban; strata); survey weights (probability weight $=$ sample weight $/ 1,000,000)$ [19]. All estimates were reported in weighted value (unless otherwise indicated) including frequency, and proportion (\%). The clustering effect of complex sampling design was adjusted using survey 'svy' set command in Stata 14.0. 
1 Before running the multivariable multinomial regression model, multicollinearity was

2 checked and excluded independent variables having variation inflation factors $\geq 3$

3 [33]. Backwards elimination multivariable multinomial logistic regression analyses were conducted [34]. First, the full multivariable regression model was run, estimated $p$-value for each independent variable. Then identified the most insignificant variable was deleted comparing $p$ values. This procedure was repeated until no insignificant independent variable was left at $p<0.2$ [35]. The statistical significance level was set $p<0.05$ (two-tailed) to identify the independent variables associated with the outcome variable. The goodness of fit test was conducted using the Log-likelihood Ratio (LR) test [10]. All analyses were conducted using Stata 14.0 (Stata Corp, 2015).

\section{Results}

\section{Background characteristics of women}

13 Table 1 shows the background characteristics of women included in this study. Among the 1,978 women, $42 \%$ were from households in the lowest two wealth quintiles. More than two-thirds (69\%) of women were from disadvantaged ethnic groups, mostly Madhesi, Janajatis and Dalits. Nearly two in five women (42\%) were native Nepali speakers (the national language). Nepali is primarily spoken in the Hill region, where most of the residents are from relatively advantaged ethnicities [36]. Male household head characterised more than two-thirds $(73 \%)$ of the households. More than half (55\%) of women were from the Terai (Plain) Region. One in four women $(26 \%)$ were from province two, whereas the smallest percentage of women $(6 \%)$ were from province six. About half (46\%) of women were from urban areas. Two-thirds (67\%) of women had no decision-making authority in relation to accessing in health-seeking, buying something (financial decision making in the family) or meeting with relatives (movement). Nearly one-third (29\%) of women reported any kind of perceived violence (e.g., beating when food burnt or beating if women went out without asking husband). In total, four in five (79.7\%) women were aged 20-34 years, and approximately $69 \%$ of women did not have a bank account.

Three in five women felt distance to a health facility was a challenge when accessing health services. Further, nearly $72 \%$ of women perceived it as challenging to access care when there was no available female healthcare worker. In addition, over twothirds (68\%) of women had no awareness of the availability of a health mothers' 
1 group in their community. One in ten mothers gave childbirth through caesarean-

2 section.

3 Table 1: Characteristics of women who had a live birth in the two years preceding the survey 4 in Nepal in NDHS 2016.

\begin{tabular}{|c|c|c|}
\hline Determinants & Categories & Frequency $(\mathrm{N}=1978)(\%)$ \\
\hline \multicolumn{3}{|l|}{ Structural } \\
\hline \multirow[t]{2}{*}{ Wealth status } & Lower (40\%) & $832(42.0)$ \\
\hline & Upper $(60 \%)$ & $1146(58.0)$ \\
\hline \multirow[t]{2}{*}{ Ethnicity } & Disadvantaged & $1374(69.5)$ \\
\hline & Advantaged & $604(30.5)$ \\
\hline \multirow[t]{2}{*}{ Religion } & Others & $306(15.5)$ \\
\hline & Hindu & $1672(84.5)$ \\
\hline \multirow[t]{3}{*}{ Maternal education } & No & $570(28.8)$ \\
\hline & Primary & $391(19.8)$ \\
\hline & $\begin{array}{l}\text { Secondary or } \\
\text { higher }\end{array}$ & $1016(51.4)$ \\
\hline \multirow[t]{3}{*}{ Maternal occupation } & Not working & $928(46.9)$ \\
\hline & Agriculture & $824(41.6)$ \\
\hline & Working paid & $227(11.5)$ \\
\hline \multirow[t]{2}{*}{ Perceived violence } & No & $1397(70.6)$ \\
\hline & Yes & $581(29.4)$ \\
\hline \multirow[t]{2}{*}{ Decision-making authority } & No & $1324(66.9)$ \\
\hline & Yes & $654(33.0)$ \\
\hline \multirow[t]{2}{*}{ Household head } & Male & $1438(72.7)$ \\
\hline & Female & $540(27.3)$ \\
\hline \multicolumn{3}{|l|}{ Intermediary } \\
\hline \multirow[t]{4}{*}{ Languages } & Nepali & $839(42.4)$ \\
\hline & Maithili & $360(18.2)$ \\
\hline & Bhojpuri & $267(13.5)$ \\
\hline & Others & $512(25.9)$ \\
\hline \multirow[t]{2}{*}{ Residence } & Urban & $1062(53.7)$ \\
\hline & Rural & $916(46.3)$ \\
\hline \multirow[t]{7}{*}{ Province } & One & $338(17.1)$ \\
\hline & Two & $513(25.9)$ \\
\hline & Three & $312(15.8)$ \\
\hline & Four & $164(8.3)$ \\
\hline & Five & $364(18.4)$ \\
\hline & Six & $121(6.1)$ \\
\hline & Seven & $166(8.4)$ \\
\hline \multirow[t]{3}{*}{ Region } & Mountain & $131(6.6)$ \\
\hline & Hills & $760(38.4)$ \\
\hline & Terai & $1087(55.0)$ \\
\hline \multirow[t]{3}{*}{ Maternal age (years) } & $15-19$ & $291(14.7)$ \\
\hline & $20-34$ & $1570(79.7)$ \\
\hline & $35+$ & $106(5.3)$ \\
\hline
\end{tabular}




\begin{tabular}{l|l|r}
\hline Determinants & Categories & Frequency (N=1978) (\%) \\
\hline Birth order (index child) & $<4$ & $1678(84.8)$ \\
\hline Sex of index child & $\geq 4$ & $300(15.2)$ \\
\hline & Male & $1063(53.7)$ \\
\hline Access to bank account & Female & $915(46.2)$ \\
\hline & No & $1367(69.1)$ \\
\hline Media exposure & Yes & $611(30.9)$ \\
\hline Last birth (index child) & No & $911(46.0)$ \\
\hline & Yes & $1067(54.0)$ \\
\hline Distance to health facilities as a & Unwanted & $418(21.1)$ \\
\hline perceived problem & Wanted & $1560(78.8)$ \\
\hline & No problem & $763(38.6)$ \\
\hline Health system & & $1213(61.4)$ \\
\hline Perceived problem not having female & Nig problem & $562(28.4)$ \\
providers & No problem & $1416(71.6)$ \\
\hline & & $1340(67.7)$ \\
\hline Awareness on health mothers' group & Big problem & $638(32.3)$ \\
\hline & Yes & $1780(90.0)$ \\
\hline Mode of delivery & Normal & $198(10.0)$ \\
\hline & Caesarean section & \\
\hline & &
\end{tabular}

2 (Dis)continuity of care of routine MNH visits in antenatal, intrapartum, and 3 postnatal period

4 Figure 2 shows the continuity of routine $\mathrm{MNH}$ visits across the CoC. Among 1,978

5 women included in this analysis, only two in five (41\%) attended all three $\mathrm{MNH}$ visits

6 (4ANC visits, institutional delivery, and one PNC visit within 48 hours of childbirth).

7 Almost all (96\%) received at least one ANC visit, but only $71 \%$ completed 4ANC

8 visits. More than one in two women (52\%) completed at least 4ANC visits and

9 received institutional delivery services. Women without 4ANC visits, however, had a

10 higher rate of home delivery. For instance, among women who were unable to

11 complete 4ANC visits, $58 \%$ of them gave birth at home, while $71 \%$ of women with no

12 ANC visits $(n=72)$ delivered at home. Only $4 \%$ (of $N=1,978)$ of women did not

13 receive any of ANC visits or institutional delivery, or PNC visit (Figure 2). 


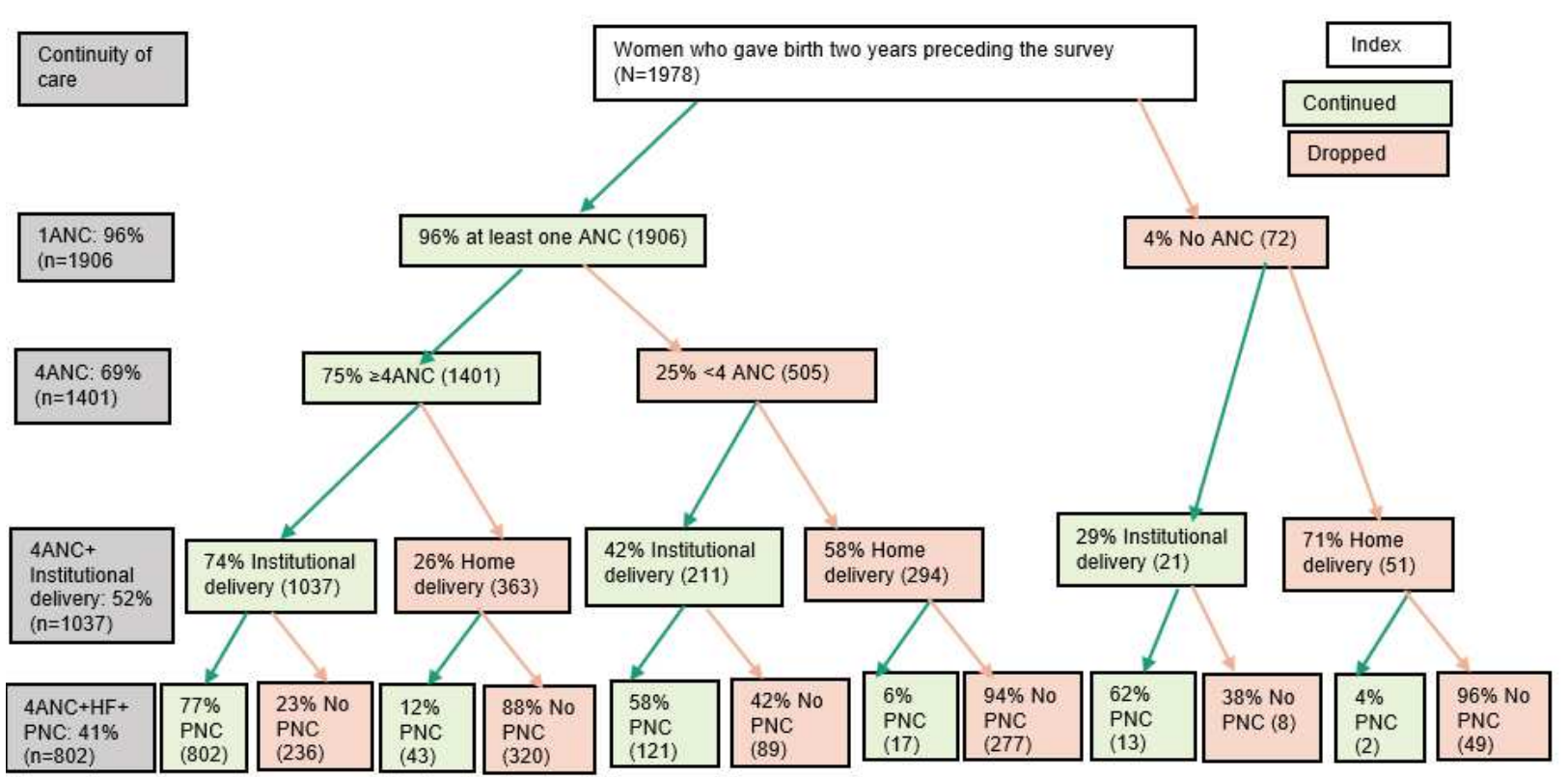

2 Figure 2: (Dis)continuity of routine $\mathrm{MNH}$ visit during the antenatal-postnatal period in Nepal, 3 NDHS 2016. Values in parenthesis indicate the number of women.

4 Table 2 shows the women who completed/discontinued MNH visits across the CoC.

5 Over half of women completed all visits if they were from provinces three $(51 \%)$ and

6 four (54\%), belonged to advantaged ethnicity (54\%), had secondary or higher-level

7 education (54\%), had jobs (53\%), had a bank account (54\%), had media exposure

8 (51\%), perceived the distance to the health facility was not a problem (53\%) and who

9 delivered via caesarean-section (71\%). However, only one in four women completed

10 all three $\mathrm{MNH}$ services if they were from province six (24\%), a Bhojpuri speaker

11 (21\%), illiterate (25\%), and higher birth order ( $\geq 4)(21 \%)$ (Table 2).

13 Table 2: (Dis)continuity of care of routine MNH visits across the CoC in Nepal, 2016.

\begin{tabular}{|c|c|c|c|c|c|c|}
\hline Determinants & Frequency & $\begin{array}{l}\text { Discontinued } \\
\text { before } \\
\text { completing } \\
\text { 4ANC visits (\%) }\end{array}$ & $\begin{array}{l}\text { Discontinued } \\
\text { before completing } \\
\text { institutional } \\
\text { delivery (\%) }\end{array}$ & $\begin{array}{l}\text { Discontinued } \\
\text { before } \\
\text { completing } \\
\text { NC visit (\%) }\end{array}$ & $\begin{array}{l}\text { Completed } \\
\text { all three } \\
\text { visits (\%) }\end{array}$ & $p$ \\
\hline Structural & 1,978 & 29.2 & 18.4 & 11.9 & 40.6 & \\
\hline \multicolumn{7}{|l|}{ Wealth status } \\
\hline Lower (40\%) & 832 & 35.1 & 26.2 & 8.6 & 30.0 & $<0.001$ \\
\hline Upper (60\%) & 1,146 & 24.8 & 12.7 & 14.3 & 48.2 & \\
\hline \multicolumn{7}{|l|}{ Ethnicity } \\
\hline Disadvantaged & 1,374 & 34.3 & 19.6 & 11.3 & 34.8 & $<0.001$ \\
\hline Advantaged & 604 & 17.6 & 15.5 & 13.4 & 53.6 & \\
\hline Religion & & & & & & \\
\hline
\end{tabular}




\begin{tabular}{|c|c|c|c|c|c|c|}
\hline Determinants & Frequency & $\begin{array}{l}\text { Discontinued } \\
\text { before } \\
\text { completing } \\
\text { 4ANC visits (\%) }\end{array}$ & $\begin{array}{l}\text { Discontinued } \\
\text { before completing } \\
\text { institutional } \\
\text { delivery (\%) }\end{array}$ & $\begin{array}{l}\text { Discontinued } \\
\text { before } \\
\text { completing } \\
\text { NC visit (\%) }\end{array}$ & $\begin{array}{l}\text { Completed } \\
\text { all three } \\
\text { visits (\%) }\end{array}$ & $p$ \\
\hline Others & 306 & 39.1 & 16.2 & 10.1 & 34.5 & 0.056 \\
\hline Hindu & 1672 & 27.3 & 18.8 & 12.2 & 41.7 & \\
\hline \multicolumn{7}{|l|}{ Maternal education } \\
\hline Illiterate & 570 & 46.5 & 20.2 & 8.8 & 24.6 & $<0.001$ \\
\hline Primary & 391 & 36.5 & 24.9 & 10.0 & 28.6 & \\
\hline Secondary or more & 1,016 & 16.6 & 14.8 & 14.4 & 54.1 & \\
\hline \multicolumn{7}{|c|}{ Maternal occupation } \\
\hline Not working & 928 & 32.9 & 13.7 & 12.9 & 40.5 & $<0.001$ \\
\hline Agriculture & 823 & 27.6 & 25.0 & 10.0 & 37.3 & \\
\hline Working paid & 227 & 19.2 & 13.6 & 14.5 & 52.7 & \\
\hline \multicolumn{7}{|l|}{ Perceived violence } \\
\hline No & 1,397 & 27.8 & 18.1 & 11.2 & 42.9 & 0.044 \\
\hline Yes & 581 & 32.4 & 19.0 & 13.7 & 34.9 & \\
\hline \multicolumn{7}{|l|}{ Decision-making } \\
\hline No & 1,324 & 30.9 & 18.9 & 11.6 & 38.6 & 0.111 \\
\hline Yes & 654 & 25.6 & 17.3 & 12.6 & 44.5 & \\
\hline \multicolumn{7}{|l|}{ Household head } \\
\hline Male & 1,438 & 30.4 & 18.9 & 12.2 & 38.6 & 0.073 \\
\hline Female & 540 & 25.9 & 17.0 & 11.2 & 45.9 & \\
\hline \multicolumn{7}{|l|}{ Intermediary } \\
\hline \multicolumn{7}{|l|}{ Language } \\
\hline Nepali & 839 & 19.8 & 17.8 & 13.2 & 49.2 & $<0.001$ \\
\hline Maithili & 360 & 32.4 & 24.5 & 11.2 & 32.0 & \\
\hline Bhojpuri & 267 & 54.4 & 12.1 & 12.2 & 21.3 & \\
\hline Others & 512 & 29.1 & 18.2 & 10.1 & 42.6 & \\
\hline \multicolumn{7}{|l|}{ Residence } \\
\hline Urban & 1,062 & 24.5 & 14.0 & 13.5 & 48.0 & $<0.001$ \\
\hline Rural & 916 & 34.6 & 23.4 & 10.0 & 32.0 & \\
\hline \multicolumn{7}{|l|}{ Provinces } \\
\hline One & 338 & 21.2 & 23.0 & 8.3 & 47.5 & $<0.001$ \\
\hline Two & 513 & 42.0 & 19.2 & 12.0 & 26.9 & \\
\hline Three & 312 & 24.2 & 14.2 & 10.7 & 50.9 & \\
\hline Four & 164 & 24.7 & 11.9 & 9.0 & 54.4 & \\
\hline Five & 364 & 25.3 & 19.5 & 13.5 & 41.7 & \\
\hline Six & 121 & 45.4 & 22.0 & 9.0 & 23.6 & \\
\hline Seven & 166 & 16.0 & 15.5 & 23.0 & 45.5 & \\
\hline \multicolumn{7}{|l|}{ Region } \\
\hline Mountain & 131 & 28.3 & 30.5 & 3.4 & 37.8 & 0.001 \\
\hline Hills & 760 & 23.8 & 18.2 & 11.7 & 46.3 & \\
\hline Terai & 1,087 & 33.0 & 17.0 & 13.1 & 36.9 & \\
\hline \multicolumn{7}{|c|}{ Maternal age (years) } \\
\hline$<19$ & 291 & 25.8 & 15.7 & 16.9 & 41.6 & 0.146 \\
\hline $20-34$ & 1,582 & 29.2 & 18.8 & 11.1 & 40.9 & \\
\hline$\geq 35$ & 106 & 37.7 & 19.7 & 10.2 & 32.4 & \\
\hline \multicolumn{7}{|l|}{ Birth order } \\
\hline$<4$ & 1,678 & 24.9 & 17.7 & 13.3 & 44.1 & $<0.001$ \\
\hline$\geq 4$ & 300 & 52.8 & 22.3 & 4.4 & 20.6 & \\
\hline \multicolumn{7}{|l|}{ Sex of index child } \\
\hline Male & 1,063 & 28.9 & 17.4 & 12.1 & 41.6 & 0.699 \\
\hline Female & 915 & 29.5 & 19.5 & 11.7 & 39.4 & \\
\hline \multicolumn{7}{|l|}{$\begin{array}{l}\text { Access to bank } \\
\text { account }\end{array}$} \\
\hline No & 1,367 & 33.9 & 20.2 & 11.6 & 34.4 & $<0.001$ \\
\hline Yes & 611 & 18.6 & 14.3 & 12.7 & 54.4 & \\
\hline \multicolumn{7}{|l|}{ Media exposure } \\
\hline No & 911 & 40.0 & 22.6 & 9.5 & 27.8 & $<0.001$ \\
\hline Yes & 1,067 & 19.9 & 14.7 & 13.9 & 51.4 & \\
\hline Last child (index & & & & & & \\
\hline
\end{tabular}




\begin{tabular}{|c|c|c|c|c|c|c|}
\hline Determinants & Frequency & $\begin{array}{l}\text { Discontinued } \\
\text { before } \\
\text { completing } \\
\text { 4ANC visits (\%) }\end{array}$ & $\begin{array}{l}\text { Discontinued } \\
\text { before completing } \\
\text { institutional } \\
\text { delivery }(\%)\end{array}$ & $\begin{array}{l}\text { Discontinued } \\
\text { before } \\
\text { completing } \\
\text { NC visit (\%) }\end{array}$ & $\begin{array}{l}\text { Completed } \\
\text { all three } \\
\text { visits (\%) }\end{array}$ & $p$ \\
\hline \multicolumn{7}{|l|}{ child) } \\
\hline Unwanted & 418 & 39.7 & 16.4 & 11.1 & 32.9 & $<0.001$ \\
\hline Wanted & 1,560 & 26.3 & 18.9 & 12.1 & 42.6 & \\
\hline \multicolumn{7}{|c|}{$\begin{array}{l}\text { Distance to health } \\
\text { facilities as a } \\
\text { perceived problem }\end{array}$} \\
\hline No problem & 763 & 23.0 & 13.6 & 12.2 & 51.3 & $<0.001$ \\
\hline Big problem & 1,215 & 33.0 & 21.4 & 11.8 & 33.8 & \\
\hline \multicolumn{7}{|l|}{ Health system } \\
\hline \multicolumn{7}{|c|}{$\begin{array}{l}\text { Perceived problem } \\
\text { not having female } \\
\text { providers }\end{array}$} \\
\hline No problem & 562 & 22.1 & 14.1 & 10.7 & 53.1 & $<0.001$ \\
\hline Big Problem & 1,416 & 31.9 & 20.1 & 12.4 & 35.6 & \\
\hline \multicolumn{7}{|c|}{$\begin{array}{l}\text { Awareness of health } \\
\text { mothers' group }\end{array}$} \\
\hline No & 1,340 & 32.5 & 17.7 & 11.5 & 38.3 & $<0.001$ \\
\hline Yes & 638 & 22.1 & 19.8 & 12.8 & 45.4 & \\
\hline \multicolumn{7}{|c|}{ Mode of delivery } \\
\hline Normal & 1,780 & 30.6 & 20.4 & 11.8 & 37.2 & $<0.001$ \\
\hline C-section & 198 & 16.1 & 0 & 13.0 & 70.9 & \\
\hline
\end{tabular}

ID: institutional delivery, HF: health facility, HMG: health mothers' group, p-values obtained from Fisher exact test. Other languages include (e.g., Tharu, Magar). Other religions include Buddha, Jain, Kirat, Christian.

2 Determinants of the (dis)continuity of MNH visits across the continuum of care

3 Along the pathway of the antenatal-postnatal period, there were three possible points

4 of discontinuation: before completing 4ANC visits, before completing institutional

5 delivery, and/or before completing a PNC visit. In the bivariable regression analysis,

6 several factors were associated with discontinuation along the pathway

7 (Supplementary file; Table 2). Associated structural factors were language, wealth

8 status, education; and intermediary factors significantly associated were the place of

9 residence, province, region, birth order, media exposure on public health issues,

10 access to a bank account, the intention of last birth, perceived problem of the long

11 distance to the health facility, and perceived violence. In addition, health system

12 factors associated significantly with the discontinuity of care were the perceived

13 problem if not having female providers in health facilities, awareness of health

14 mothers' groups, and mode of delivery.

15 Table 3 shows the multivariate multinomial regression analysis of factors associated

16 with discontinuity of care during the antenatal-postnatal period. Eight determinants

17 were significantly associated with discontinuity of care before completing 4ANC

18 visits. Structural factors, such as illiteracy (Relative Risk Ratio $(R R)=2.65 ; 95 \% \mathrm{Cl}$ :

$191.72,4.08)$, lower wealth status ( $R R=2.39 ; 95 \% \mathrm{Cl}: 1.63,3.51)$, Bhojpuri language 
$1 \quad(R R=3.28 ; 95 \% \mathrm{Cl}: 1.26,8.58)$, and living in province six $(R R=4.08 ; 95 \% \mathrm{Cl}: 2.30$,

2 7.21) had a higher risk of discontinuity of care before completing 4ANC visits compared to their reference category (completing all $\mathrm{MNH}$ visits). Intermediary factors, such as women with higher birth order $(\geq 4)(R R=2.15 ; 95 \% \mathrm{Cl}: 1.41,3.30)$, women not having media exposure ( $R R=1.81 ; 95 \% \mathrm{Cl}: 1.33,2.46)$, and unwanted last birth ( $R R=2.11 ; 95 \% \mathrm{Cl}: 1.47,3.02)$ had a higher relative risk of discontinuing before completing 4ANC visits compared to their reference counterparts. Women who had no awareness of the availability of a mothers' group in their community had a higher risk (RR=1.53; $95 \% \mathrm{Cl}: 1.13,2.07)$ of discontinuing before completing 4ANC visits compared to the reference category.

Nine determinants were significantly associated with continuity of care until 4ANC visits but discontinued before completing institutional delivery (Table 3). Women who had primary education $(\mathrm{RR}=1.92 ; 95 \% \mathrm{Cl}: 1.26,2.93)$ and lower wealth status $(R R=2.82 ; 95 \% \mathrm{Cl}: 1.88,4.22)$, who were involved in agriculture ( $R R=1.51 ; 95 \%$ $\mathrm{Cl}: 1.04,2.19)$ or from disadvantaged ethnicity $(\mathrm{RR}=1.54 ; 95 \% \mathrm{Cl}: 1.05,2.26)$ had a higher relative risk of discontinuity of care before completing institutional delivery (compared to the continuity of care of all $\mathrm{MNH}$ visits) compared to women with secondary or higher education, higher wealth status, women who had paid jobs, and women of advantaged ethnicities, respectively. Moreover, women who lived in rural areas ( $R R=1.91 ; 95 \% \mathrm{Cl}: 1.36,2.69)$ had a higher risk of discontinuity of care before completing institutional delivery compared to women from urban areas. Additionally, women with no media exposure (e.g. radio, newspaper, television) ( $R R=1.56 ; 95 \%$ $\mathrm{Cl}: 1.13,2.14)$ had a higher risk of continuity of 4 ANC visits. However, women from province four ( $R R=0.47 ; 95 \% \mathrm{Cl}: 0.23,0.94)$ and those aged $15-19$ years $(R R=0.63$; $95 \% \mathrm{Cl}: 0.39,0.99)$ had significantly associated with a lower risk of discontinuation before completing institutional delivery (compared to completion of all three $\mathrm{MNH}$ visits) compared to women from province one and those aged 20-34 years.

Two determinants were associated with continuity of care until 4ANC visits and institutional delivery but discontinued before completing PNC visits (Table 3). Women from provinces six $(R R=2.24 ; 95 \% \mathrm{Cl}: 1.07,4.71)$ and seven $(R R=3.57 ; 95 \% \mathrm{Cl}$ : $1.87,6.81$ ), and women with a perceived problem of not having a female provider $(\mathrm{RR}=1.64 ; 95 \% \mathrm{Cl}: 1.12,2.39)$ had a higher risk of completing all 4ANC visits and 
1 institutional delivery compared to their respective reference category (completion of 2 all routine $\mathrm{MNH}$ visits). 
1 Table 3: Multivariale multinomial logistic regression of determinants of continuity of routine MNH visits in Nepal, NDHS 2016 (N=1,978)

\begin{tabular}{|c|c|c|c|}
\hline Determinants & $\begin{array}{l}\text { Discontinued before completing } \\
\text { 4ANC visits (ARR; } 95 \% \mathrm{Cl} \text { ) }\end{array}$ & $\begin{array}{l}\text { Discontinued before completing } \\
\text { institutional delivery (ARR; } 95 \% \mathrm{Cl} \text { ) }\end{array}$ & $\begin{array}{l}\text { Discontinued before completing PNC } \\
\text { visit (ARR;95\% Cl) }\end{array}$ \\
\hline \multicolumn{4}{|l|}{ Structural } \\
\hline \multicolumn{4}{|l|}{ Wealth status } \\
\hline Upper (60\%) & 1.00 & 1.00 & 1.00 \\
\hline Lower (40\%) & $2.39(1.63,3.51)^{\star \star \star}$ & $2.82(1.88,4.22)$ *** & $0.93(0.59,1.48)$ \\
\hline \multicolumn{4}{|l|}{ Ethnicity } \\
\hline Advantaged & 1.00 & 1.00 & 1.00 \\
\hline Disadvantaged & $1.47(0.79,2.71)$ & $1.54(1.05,2.26)$ * & $1.28(0.82,1.98)$ \\
\hline \multicolumn{4}{|c|}{ Maternal occupation } \\
\hline Agriculture & $0.79(0.56,1.11)$ & $1.51(1.04,2.19)^{*}$ & $0.87(0.59,1.29)$ \\
\hline Housewife & 1.00 & 1.00 & 1.00 \\
\hline Working paid & $0.64(0.35,1.15)$ & $1.22(0.70,2.13)$ & $1.08(0.62,1.90)$ \\
\hline \multicolumn{4}{|c|}{ Maternal education } \\
\hline Higher & 1.00 & 1.00 & 1.00 \\
\hline Illiterate & $2.65(1.72,4.08)^{* \star *}$ & $1.39(0.93,2.08)$ & $1.21(0.72,2.02)$ \\
\hline Primary & $2.41(1.62,3.57)^{* * *}$ & $1.92(1.26,2.93)$ ** & $1.20(0.74,1.93)$ \\
\hline \multicolumn{4}{|l|}{ Intermediary } \\
\hline \multicolumn{4}{|l|}{ Language } \\
\hline Nepali & 1.00 & 1.00 & 1.00 \\
\hline Maithili & $1.31(0.57,3.00)$ & $1.36(0.6,3.11)$ & $0.71(0.29,1.77)$ \\
\hline Bhojpuri & $3.28(1.26,8.58)$ * & $0.97(0.35,2.68)$ & $1.06(0.44,2.57)$ \\
\hline Others & $1.52(0.81,2.84)$ & $0.89(0.55,1.42)$ & $0.66(0.41,1.05)$ \\
\hline \multicolumn{4}{|l|}{ Province } \\
\hline One & 1.00 & 1.00 & 1.00 \\
\hline Two & $1.73(0.93,3.21)$ & $1.34(0.67,2.71)$ & $2.19(0.89,5.42)$ \\
\hline Three & $1.31(0.68,2.54)$ & $0.75(0.41,1.35)$ & $1.14(0.57,2.27)$ \\
\hline Four & $1.45(0.79,2.68)$ & $0.47(0.23,0.94)$ * & $0.95(0.46,1.97)$ \\
\hline Five & $1.10(0.61,1.96)$ & $1.09(0.67,1.76)$ & $1.93(0.98,3.82)$ \\
\hline Six & $4.08(2.30,7.21)^{* * *}$ & $1.32(0.65,2.68)$ & $2.24(1.07,4.71)^{*}$ \\
\hline Seven & $0.56(0.29,1.07)$ & $0.51(0.25,1.02)$ & $3.57(1.87,6.81)^{* * *}$ \\
\hline \multicolumn{4}{|l|}{ Residence } \\
\hline Urban & 1.00 & 1.00 & 1.00 \\
\hline
\end{tabular}




\begin{tabular}{|c|c|c|c|}
\hline Determinants & $\begin{array}{l}\text { Discontinued before completing } \\
\text { 4ANC visits (ARR; } 95 \% \mathrm{Cl} \text { ) }\end{array}$ & $\begin{array}{l}\text { Discontinued before completing } \\
\text { institutional delivery (ARR; } 95 \% \mathrm{Cl} \text { ) }\end{array}$ & $\begin{array}{l}\text { Discontinued before completing PNC } \\
\text { visit (ARR;95\% Cl) }\end{array}$ \\
\hline Rural & $1.35(0.95,1.93)$ & $1.91(1.36,2.69)^{* * *}$ & $0.97(0.64,1.48)$ \\
\hline \multicolumn{4}{|c|}{ Maternal age (in years) } \\
\hline $15-19$ & $0.79(0.52,1.20)$ & $0.63(0.39,0.99)$ * & $1.24(0.78,1.95)$ \\
\hline $20-34$ & 1.00 & 1.00 & 1.00 \\
\hline 35 or above & $0.55(0.29,1.04)$ & $0.79(0.35,1.78)$ & $1.66(0.67,4.11)$ \\
\hline \multicolumn{4}{|l|}{ Birth order } \\
\hline$<4$ & 1.00 & 1.00 & 1.00 \\
\hline$\geq 4$ & $2.15(1.41,3.30)^{* * *}$ & $1.5(0.98,2.30)$ & $0.52(0.26,1.06)$ \\
\hline \multicolumn{4}{|c|}{ Media exposure } \\
\hline Yes & 1.00 & 1.00 & 1.00 \\
\hline No & $1.81(1.33,2.46)^{* * *}$ & $1.56(1.13,2.14)^{* *}$ & $1.01(0.69,1.50)$ \\
\hline \multicolumn{4}{|c|}{ Last birth (index child) } \\
\hline Wanted & 1.00 & 1.00 & 1.00 \\
\hline Unwanted & $2.11(1.47,3.02)^{* \star *}$ & $1.15(0.76,1.72)$ & $1.10(0.67,1.82)$ \\
\hline \multicolumn{4}{|l|}{ Health system } \\
\hline \multicolumn{4}{|c|}{$\begin{array}{l}\text { Perceived problem not having female } \\
\text { providers }\end{array}$} \\
\hline No problem & 1.00 & 1.00 & 1.00 \\
\hline Big problem & $1.25(0.89,1.76)$ & $1.46(1.05,2.04)^{*}$ & $1.64(1.12,2.39)$ * \\
\hline \multicolumn{4}{|c|}{ Awareness on health mothers' group } \\
\hline Yes & 1.00 & 1.00 & 1.00 \\
\hline No & $1.53(1.13,2.07)$ ** & $1.10(0.79,1.53)$ & $1.06(0.76,1.46)$ \\
\hline
\end{tabular}

${ }^{* * *} \mathrm{p}<0.001,{ }^{* *} \mathrm{p}<0.01,{ }^{*} \mathrm{p}<0.05$. Determinants that had $\mathrm{p}<0.2$ included in the final model adjusting for covariates listed in the table. The likelihood ratio of the reduced model with the full model was [chi-square=19.47; $\mathrm{p}=0.0 .555$ ], and our model was the best fit. HWs: health workers, HMG: health mothers' group, HF: health facility, ANC: antenatal care. The reference category of outcome variable was the completion of all three MNH visits. Other languages include Tharu, Magar. ARR: Adjusted risk ratio. 


\section{Discussion}

2 The current study examined the composite of coverage routine $\mathrm{MNH}$ visits and had

3 low completion of all routine $\mathrm{MNH}$ visits across the $\mathrm{CoC}$. We found more than two-in-

4 five $(41 \%)$ women in Nepal received all routine MNH visits across the CoC. There

5 was high proportion of discontinuation around later weeks of pregnancy (4ANC visits) or around childbirth (institutional delivery). Several structural determinants were found to be associated with discontinuity of care across the CoC. For instance, women with structural disadvantages (e.g., disadvantaged ethnicity, women of lower wealth status, illiterate women) had a higher risk of discontinuation across the CoC. Intermediary and health system determinants contributed to the (dis)continuity of care of MNH services (e.g., provinces six or seven, Maithali speaker women, high birth order, and poor media exposure on health issues); if women had poor awareness on health mothers' group, and perceived problem if not having female providers in health facility found to have higher risk of discontinuation of $\mathrm{MNH}$ visits across the CoC.

The reasons for low $\mathrm{CoC}$ in this study may be due to high discontinuation around later gestational week of childbirth, and no PNC visit of women gave birth in health facilities. Women delivering in health facilities should receive at least one PNC visit within 48 hours (at least at the time of discharge from health facilities after childbirth), but the lower PNC visit compared to institutional delivery, suggests not all women/newborns received PNC visit even where delivery took place in a healthcare facility. The lower completion of all routine $\mathrm{MNH}$ visits was consistent with the studies in Cambodia [37] and Lao PDR [38]. Other studies have reported poor uptake of $4 A N C$ visits and institutional delivery due to long waking hours, and unavailability of necessary arrangements in local health facilities [39, 40]. In later weeks prior to childbirth or around childbirth, pregnant women may face difficulties to reach healthcare facilities if physical access is poor, or there is no suitable accommodation close to the health facility [42]. A study in Nepal also indicated that despite the accessibility of healthcare facilities, knowledge of and demand for PNC was low [43]. The accrediting health posts to birthing centers and strengthening existing birthing could increase the availability of intrapartum care in rural health facilities. On the other hand, ensuring necessary arrangement to reach health 
1 facilities could increase institutional delivery and first PNC visit within 48 hours of

2 childbirth. In the interim, the provision of trained community health workers could

3 increase the utilisation of PNC.

4

5 The current study revealed women with social disadvantages (illiterate, poor,

6 marginalised ethnic group, involved in agricultural work) and geographical factors

7 had higher discontinuation across the CoC. Other studies have reported women's

8 living, and working life [40] also influences the uptake of maternity services [44], and

9 women with poor wealth status have poor MNH coverage during pregnancy and childbirth [44]. Such women may not seek health services unless there are complications. In addition, women with social disadvantages experience difficulties in daily life, usually have more focus on livelihood support than healthcare; they have inequitable distribution of livelihood opportunities and resources that contribute to poor access to and higher discontinuation of routine $\mathrm{MNH}$ visits. These factors are mostly non-modifiable and often require long term sociopolitical interventions [45-48], and technical and biomedical focussed approaches on their own may not improve $\mathrm{MNH}$ visits across the $\mathrm{CoC}$ [47]. Longer-term structural interventions to improve $\mathrm{MNH} \mathrm{CoC}$ may include improving female access to formal and informal education and employment opportunities [45].

Health system factors could improve the continuity of care of MNH visits across the $\mathrm{CoC}$, for instance, birth spacing, awareness on health issues through mass media exposure and having female providers at the health facility. In the current study, women who have already more than four children, or if last birth intended, such women discontinued the MNH visits in the last birth. This suggests if women had intended pregnancy, they could get priority for needed MNH visits. Effective uptake of family planning services could help for wanted pregnancy and reduced birth spacing, resulting in women could complete all $\mathrm{MNH}$ visits for their intended birth [45].

Health awareness on the importance of pregnancy, childbirth, and PNC services can be improved via exposure to mass media (e.g., local radios, television) and dissemination of health information to current and future mothers. A past study in

34 Nepal reported mass media exposure was positively associated with maternal 
1 healthcare utilisation [49]. Health awareness through mobile technology could play

2 an important role in the utilisation of health services generally [50] and the MNH services particularly [51]. Context-specific strategies can be adopted to increase the uptake of needed $\mathrm{MNH}$ visits that include outreach clinics in remote and underprivileged communities, or mobilisation of local community workers for PNC home visits [52]. In addition, properly functional health mothers' group in the community could raise awareness among pregnant women and provide necessary health information in their pregnancy and childbirth. Health mothers' groups are women-led community health groups where current and future mothers can gather and discuss reproductive, maternal, child health and nutrition issues [53]. Such health groups could address the social taboos as talking about reproductive healthrelated issues is culturally taboo in Nepali society, and women are usually like to share the provision of female providers [26, 54]. Additionally, the current SDIP has provisioned 4ANC visits and institutional delivery, but the provision of financial incentive lacks for PNC visit. Ensuring financial incentive in the SDIP programs could increase the uptake of all three $\mathrm{MNH}$ visits.

The measurement of continuity of care is important for tracking health services coverage across the $\mathrm{MNH}$ continuum essential by creating a composite coverage indicator of all three routine MNH visits. Such measurement and tracking could give the actual coverage of MNH visits across the CoC. Past studies $[55,56]$ and routine monitoring system [57] lack measurement of composite coverage of all routine $\mathrm{MNH}$ visits across the $\mathrm{CoC}$. The completion of all $\mathrm{MNH}$ visits is a critical window of opportunity to receive recommended $\mathrm{MNH}$ interventions for the survival of mothers and newborns. A modelling study estimated that increased access and quality of MNH interventions across the CoC could avert up to $71 \%$ and $54 \%$ neonatal and maternal deaths respectively, and $33 \%$ of stillbirths annually [5]. The composite coverage measurement of $\mathrm{MNH}$ visits can have a significant implication in countries with high maternal and neonatal deaths.

\section{Policy and programmatic implications}

This study has some implications for programs and research. First, the creation and execution of a composite indicator provided insight into $\mathrm{MNH} \mathrm{CoC}$ and should be included in routine health management information system (HMIS) and periodic 
1 health survey (e.g., demographic and health survey). The quality of MNH services is

2 prioritised in SDG3, which focuses on universal coverage of MNH services [58].

3 Universal coverage of quality MNH services is crucial for better $\mathrm{MNH}$ outcomes and

4 in achieving SDG3. The government of Nepal should focus its programs targeting women living in difficult geographical areas (e.g., province six) and women with social disadvantages (e.g., poor, marginalised ethnicity). Supply-side approaches found to improve the better health services delivery included strengthening birthing center (e.g., health logistics, human resources and training), and establishing maternity waiting home [59]. Availability of childbirth services in all rural health facilities could increase the facility childbirth and first PNC visit. The home visits through community health workers in hard to reach community could also improve PNC visit home visit.

Second, the provision of female health providers could improve the counselling on the importance of $\mathrm{MNH}$ services in the $\mathrm{MNH}$ continuum. Other potential strategies could be mass media mobilisation to raise awareness on the $\mathrm{COC}$ and focused $\mathrm{MNH}$ services to disadvantaged population groups, such as women of lower wealth status, who speak Maithali speak, living in remote areas. The health system could formulate focussed service delivery packages to women with structural disadvantages, for instance, provision of focus incentive to those groups who are most marginalised women, Dalits, Karnali province, Maithali speaking women. The current safe delivery incentive program (SDIP) includes separate incentives for 4ANC visits, and institutional delivery program [60]. This SDIP should be reformed by providing incentive for women those who complete 4ANC visits, delivered babies in health facilities and complete first PNC visit. Additionally, such incentive program needs to be designed for specific groups based on marginalisation status. The composite coverage indicator employed in this study could help to reform the SDIP.

Third, health services need to focus on remote areas, including improving family planning services. Better family planning services could improve the birth spacing, thereby reducing unintended pregnancy and reduced numbers of parity. Women of wanted pregnancy may complete all routine $\mathrm{MNH}$ visits and receive all essential antenatal, intrapartum, and postnatal interventions for their and newborns better 34 health. 


\section{Strengths and limitations of the study}

3 This study has some strengths and limitations. Strengths included; first, this study is based on a nationally representative survey with higher response rate (98\%), and findings could be generalised at the national level. Second, this study considered the PNC visit for mothers and newborns rather than previous studies that examined PNC visit for newborn or PNC mothers separately. This study has the following limitations.

8 First, inferences drawn from this study are based on an observational and crosssectional design, which allows the study of correlations rather than causality. Second, the NDHS 2016 collected information based on recall of women who had a live birth five years prior to the survey (2011-2016); however, we included a short recall period of two years restricting study sample of women who had a live birth two years preceding the survey (2014-2016). Third, this study is based on secondary data analysis; we were unable to include important variable such as obstetric complications that could contribute to discontinuation along the pathway. Fourth, the outcome variable was self-reported after face-to-face interviews with women, which may have social desirability bias (e.g., over-reporting of good behaviours and underreporting of bad behaviours) and misclassification. Finally, from the research perspective, this study has not explored stories of why women discontinued health services utilisation across the CoC. The qualitative study could provide a deeper understanding of real stories of the underlying reasons for discontinuation across the CoC.

\section{Conclusions}

Only two-in-five women completed all MNH visits across the CoC. Disadvantaged women had high discontinuation in different stages of $\mathrm{CoC}$. Creation and execution of composite coverage of 4ANC visits, institutional delivery and PNC visits could track the uptake of health services across the $\mathrm{CoC}$. Monitoring of health services utilisation using composite coverage indicator and provision of focused strategies (e.g., home visits and outreach services, incentive who complete all MNH visits) could increase the completion of all $\mathrm{MNH}$ visits across the $\mathrm{CoC}$, especially among disadvantaged women. The provision of continuous availability of $\mathrm{MNH}$ services in health facilities, trained female health services providers, and provision of quality

34 health care could improve the continuity of care during pregnancy, intrapartum, and 
1 the postnatal period.

2 Supplementary file

3 Table 1: Description of variables included in the analysis of (dis)continuity of care of

4 routine $\mathrm{MNH}$ visits in Nepal, 2016

5 Table 2: Bivariable multinomial logistic regression analysis

6 Abbreviations

7 ANC: Antenatal Care; CoC: Continuum of care; LMICs: Low and lower-middle-

8 income countries; MNH: Maternal and newborn health; NDHS: Nepal Demographic

9 and Health Survey; PNC: Postnatal Care; SDIP: Safe Delivery Incentive Program

\section{Declaration}

12 Ethics approval and consent to participate

13 We used secondary data from the NDHS 2016. The NDHS 2016 was approved by

14 an ethical review board of Nepal Health Research Council, Nepal, and ICF Marco

15 International, Maryland, USA. The Ministry of Health and Population (MOHP)

16 (Nepal), ICF International Maryland, and DHS program (USA) approved and

17 oversaw the overall research process of the NDHS 2016. The NDHS data are

18 publicly available for further analysis, and data were deidentified of the research

19 participants. This study did not require ethical approval from respective institutions.

20 However, the first author took approval for the download and use of dataset for his

21 doctoral thesis, downloaded data of individual women record from the Nepal data file

22 available from the DHS program (https://dhsprogram.com/data/available-

23 datasets.cfm).

25 Consent for publication

26 Not applicable

28 Availability of data and materials

29 Data used in this study are publicly available secondary data obtained from the DHS

30 (https://dhsprogram.com/data/available-datasets.cfm) program.

32 Competing interests

33 The authors declared that they have no competing interests. 
2 No funding received for the development of this manuscript.

\section{Acknowledgements}

This manuscript is a part of the first author's doctoral thesis at the School of Public Health, the University of Queensland, supported by the University of Queensland International Scholarship and Career Development Extension Scholarship, and Research and Training Program Scholarship. The author would like to acknowledge the University of Queensland for providing the scholarship for the doctoral program. I would like to acknowledge Melinda Protani and Elisabeth Winkler for data analysis.

\section{Authors' contributions}

RBK conceived of the study, performed the statistical analysis. RBK and JD drafted the manuscript. RK, JD and YA supervised the study. All authors contributed significantly during the revision and finalised the manuscript. All authors read and agreed on the final version of the manuscript.

\section{References}

1. WHO: Trends in maternal mortality 2000 to 2017: estimates by WHO, UNICEF, UNFPA, World Bank Group and the United Nations Population Division. In. WHO Geneva,; 2019.

2. Darmstadt GL: Global perinatal health: accelerating progress through innovations, interactions, and interconnections. Semin Perinatol 2010, 34(6):367-370.

3. Darmstadt GL, Walker N, Lawn JE, Bhutta ZA, Haws RA, Cousens S: Saving newborn lives in Asia and Africa: cost and impact of phased scale-up of interventions within the continuum of care. Health Policy Plan 2008, 23(2):101-117.

4. WHO: WHO recommendations on postnatal care of the mother and newborn. In. Geneva; 2014.

5. Bhutta ZA, Das JK, Bahl R, Lawn JE, Salam RA, Paul VK, Sankar MJ, Blencowe $\mathrm{H}$, Rizvi A, Chou VB et al: Can available interventions end preventable deaths in mothers, newborn babies, and stillbirths, and at what cost? Lancet 2014, 384(9940):347-370.

6. Wang W, Hong R: The continuum of care for maternal and newborn health in Cambodia: where are the gaps and why? A population-based study. The Lancet 2013, 381:S145.

7. Kerber KJ, de Graft-Johnson JE, Bhutta ZA, Okong P, Starrs A, Lawn JE: Continuum of care for maternal, newborn, and child health: from slogan to service delivery. Lancet 2007, 370(9595):1358-1369. 
8. Chalise B, Chalise M, Bista B, Pandey AR, Thapa S: Correlates of continuum of maternal health services among Nepalese women: Evidence from Nepal Multiple Indicator Cluster Survey. PLoS One 2019, 14(4):e0215613.

9. Shibanuma A, Yeji F, Okawa S, Mahama E, Kikuchi K, Narh C, Enuameh Y, Nanishi K, Oduro A, Owusu-Agyei S et al: The coverage of continuum of care in maternal, newborn and child health: a cross-sectional study of woman-child pairs in Ghana. BMJ Glob Health 2018, 3(4):e000786.

10. Yeji F, Shibanuma A, Oduro A, Debpuur C, Kikuchi K, Owusu-Agei S, Gyapong M, Okawa S, Ansah E, Asare GQ et al: Continuum of care in a maternal, newborn and child health program in Ghana: Low completion rate and multiple obstacle factors. PLoS One 2015, 10(12):e0142849.

11. Mohan D, LeFevre AE, George A, Mpembeni R, Bazant E, Rusibamayila N, Killewo J, Winch PJ, Baqui AH: Analysis of dropout across the continuum of maternal health care in Tanzania: findings from a cross-sectional household survey. Health Policy Plan 2017, 32(6):791-799.

12. Iqbal S, Maqsood S, Zakar R, Zakar MZ, Fischer F: Continuum of care in maternal, newborn and child health in Pakistan: analysis of trends and determinants from 2006 to 2012. BMC Health Serv Res 2017, 17(1):189.

13. Henriksson DK, Fredriksson M, Waiswa P, Selling K, Swartling Peterson S: Bottleneck analysis at district level to illustrate gaps within the district health system in Uganda. Glob Health Action 2017, 10(1):1327256.

14. Singh K, Story WT, Moran AC: Assessing the continuum of care pathway for maternal health in South Asia and Sub-Saharan Africa. Matern Child Health J 2016, 20(2):281-289.

15. SDG 3: Ensure healthy lives and promote wellbeing for all at all ages [https://www.who.int/sdg/targets/en/]

16. Fullman N, Barber RM, Abajobir AA, Abate KH, Abbafati C, Abbas KM, AbdAllah F, Abdulkader RS, Abdulle AM, Abera SF et al: Measuring progress and projecting attainment on the basis of past trends of the healthrelated Sustainable Development Goals in 188 countries: an analysis from the Global Burden of Disease Study 2016. Lancet 2017, 390(10100):1423-1459.

17. Gage AD, Carnes F, Blossom J, Aluvaala J, Amatya A, Mahat K, Malata A, Roder-DeWan S, Twum-Danso N, Yahya T et al: In Low- And Middle-Income Countries, Is Delivery In High-Quality Obstetric Facilities Geographically Feasible? Health Aff (Millwood) 2019, 38(9):1576-1584.

18. Akseer N, Kamali M, Arifeen SE, Malik A, Bhatti Z, Thacker N, Maksey M, D'Silva $H$, da Silva IC, Bhutta ZA: Progress in maternal and child health: how has South Asia fared? BMJ 2017, 357:j1608.

19. MOHP [Nepal]: Nepal demographic and health survey 2016. In. Kathmandu; 2017.

20. Montagu D, Sudhinaraset M, Diamond-Smith N, Campbell O, Gabrysch S, Freedman L, Kruk ME, Donnay F: Where women go to deliver: understanding the changing landscape of childbirth in Africa and Asia. Health Policy Plan 2017, 32(8):1146-1152.

21. Shahabuddin A, De Brouwere V, Adhikari R, Delamou A, Bardaj A, Delvaux T: Determinants of institutional delivery among young married women in Nepal: Evidence from the Nepal Demographic and Health Survey, 2011. BMJ open 2017, 7(4). 
22. Moyer CA, Mustafa A: Drivers and deterrents of facility delivery in subSaharan Africa: a systematic review. Reprod Health 2013, 10(1):40.

23. Dhakal S, Chapman GN, Simkhada PP, van Teijlingen ER, Stephens J, Raja $A E$ : Utilisation of postnatal care among rural women in Nepal. $B M C$ Pregnancy Childbirth 2007, 7:19.

24. Khanal V, Adhikari M, Karkee R, Gavidia T: Factors associated with the utilisation of postnatal care services among the mothers of Nepal: analysis of Nepal demographic and health survey 2011. BMC Womens Health 2014, 14(1):19.

25. Joshi C, Torvaldsen S, Hodgson R, Hayen A: Factors associated with the use and quality of antenatal care in Nepal: a population-based study using the demographic and health survey data. BMC Pregnancy Childbirth 2014, 14:94.

26. Sharma S, van Teijlingen E, Hundley V, Angell C, Simkhada P: Dirty and $\mathbf{4 0}$ days in the wilderness: Eliciting childbirth and postnatal cultural practices and beliefs in Nepal. BMC Pregnancy Childbirth 2016, 16(1):147.

27. Conway K, Akseer N, Subedi RK, Brar S, Bhattarai B, Dhungana RR, Islam $\mathrm{M}$, Mainali $\mathrm{A}$, Pradhan $\mathrm{N}$, Tasic $\mathrm{H}$ et al: Drivers of stunting reduction in Nepal: a country case study. Am J Clin Nutr 2020.

28. Mahato PK, Waithaka E, van Teijlingen E, Pant PR, Biswas A: Social autopsy: a potential health-promotion tool for preventing maternal mortality in low-income countries. WHO South East Asia J Public Health 2018, 7(1):24-28.

29. WHO: A conceptual framework for action on the social determinants of health. In. Geneva: World Health Organization; 2010.

30. Karkee R, Lee AH, Khanal V: Need factors for utilisation of institutional delivery services in Nepal: an analysis from Nepal Demographic and Health Survey, 2011. BMJ Open 2014, 4(3):e004372.

31. Adewuyi EO, Auta A, Khanal V, Bamidele OD, Akuoko CP, Adefemi K, Tapshak SJ, Zhao Y: Prevalence and factors associated with underutilisation of antenatal care services in Nigeria: A comparative study of rural and urban residences based on the 2013 Nigeria demographic and health survey. PLoS One 2018, 13(5):e0197324.

32. Khatri RB, Poudel S, Ghimire PR: Factors associated with unsafe abortion practices in Nepal: Pooled analysis of the 2011 and 2016 Nepal Demographic and Health Surveys. PLoS One 2019, 14(10):e0223385.

33. Akinwande MO, Dikko HG, Samson A: Variance Inflation Factor: As a Condition for the Inclusion of Suppressor Variable(s) in Regression Analysis. Open Journal of Statistics 2015, 05(07):754-767.

34. Heinze G, Wallisch C, Dunkler D: Variable selection - A review and recommendations for the practicing statistician. Biom J 2018, 60(3):431449.

35. Bursac Z, Gauss CH, Williams DK, Hosmer DW: Purposeful selection of variables in logistic regression. Source Code Biol Med 2008, 3:17.

36. CBS [Nepal]: National population and housing census 2011. In.; 2012.

37. Wang W, Hong R: Levels and determinants of continuum of care for maternal and newborn health in Cambodia-evidence from a populationbased survey. BMC Pregnancy Childbirth 2015, 15(1):62. 
38. Sakuma S, Yasuoka J, Phongluxa K, Jimba M: Determinants of continuum of care for maternal, newborn, and child health services in rural Khammouane, Lao PDR. PLoS One 2019, 14(4):e0215635.

39. Khatri RB, Dangi TP, Gautam R, Shrestha KN, Homer CSE: Barriers to utilisation of childbirth services of a rural birthing center in Nepal: A qualitative study. PLoS One 2017, 12(5):e0177602.

40. Byrne A, Hodge A, Jimenez-Soto E, Morgan A: Looking beyond supply: a systematic literature review of demand-side barriers to health service utilisation in the mountains of Nepal. Asia Pac J Public Health 2013, 25(6):438-451.

41. Hodge A, Byrne A, Morgan A, Jimenez-Soto E: Utilisation of health services and geography: deconstructing regional differences in barriers to facility-based delivery in Nepal. Matern Child Health J 2015, 19(3):566-577.

42. Khatri RB, Karkee R: Social determinants of health affecting utilisation of routine maternity services in Nepal: a narrative review of the evidence. Reprod Health Matters 2018, 26(54):32-46.

43. Xu S, Blavo C, Marahatta SB, Banjara M, Amatya R, Hardigan P, Anderson N: Assessment of Healthcare Utilization among Women Experiencing Childbirth in Suburban Nepal. Journal of Health Promotion 2019, 7:53-64.

44. Devkota B, Maskey J, Pandey AR, Karki D, Godwin P, Gartoulla P, Mehata S, Aryal KK: Determinants of home delivery in Nepal - A disaggregated analysis of marginalised and non-marginalised women from the 2016 Nepal Demographic and Health Survey. PLoS One 2020, 15(1):e0228440.

45. Hamal M, Dieleman M, De Brouwere V, de Cock Buning T: Social determinants of maternal health: a scoping review of factors influencing maternal mortality and maternal health service use in India. Public Health Rev 2020, 41(1):13.

46. Mumtaz Z, Salway S, Shanner L, Bhatti A, Laing L: Maternal deaths in Pakistan: intersection of gender, caste, and social exclusion. BMC Int Health Hum Rights 2011, 11 Suppl 2(2):S4.

47. Mumtaz Z, Salway S, Bhatti A, Shanner L, Zaman S, Laing L, Ellison GT: Improving maternal health in Pakistan: toward a deeper understanding of the social determinants of poor women's access to maternal health services. Am J Public Health 2014, 104 Suppl 1(S1):S17-24.

48. Ruiz-Cantero MT, Guijarro-Garvi M, Bean DR, Martinez-Riera JR, FernandezSaez J: Governance commitment to reduce maternal mortality. A political determinant beyond the wealth of the countries. Health Place 2019, 57:313-320.

49. Fatema K, Lariscy JT: Mass media exposure and maternal healthcare utilisation in South Asia. SSM Popul Health 2020, 11:100614.

50. Willcox M, Moorthy A, Mohan D, Romano K, Hutchful D, Mehl G, Labrique A, LeFevre A: Mobile technology for community health in Ghana: is maternal messaging and provider use of technology cost-effective in improving maternal and child health outcomes at scale? J Med Internet Res 2019, 21(2):e11268.

51. Jain AK, Winfrey W: Contribution of Contraceptive Discontinuation to Unintended Births in 36 Developing Countries. Stud Fam Plann 2017, 48(3):269-278.

52. Perry HB, Sacks E, Schleiff M, Kumapley R, Gupta S, Rassekh BM, Freeman $P A$ : Comprehensive review of the evidence regarding the effectiveness 
of community-based primary health care in improving maternal, neonatal and child health: 6 . strategies used by effective projects. J Glob Health 2017, 7(1):010906.

53. Panday S, Bissell P, van Teijlingen E, Simkhada P: The contribution of female community health volunteers (FCHVs) to maternity care in Nepal: a qualitative study. BMC Health Serv Res 2017, 17(1):623.

54. Mattebo M, Bogren M, Brunner N, Dolk A, Pedersen C, Erlandsson K: Perspectives on adolescent girls' health-seeking behaviour in relation to sexual and reproductive health in Nepal. Sexual \& Reproductive Healthcare 2019, 20:7-12.

55. Shah R, Rehfuess EA, Paudel D, Maskey MK, Delius M: Barriers and facilitators to institutional delivery in rural areas of Chitwan district, Nepal: a qualitative study. Reprod Health 2018, 15(1):110.

56. Arsenault C, Jordan K, Lee D, Dinsa G, Manzi F, Marchant T, Kruk ME: Equity in antenatal care quality: an analysis of 91 national household surveys. Lancet Glob Health 2018, 6(11):e1186-e1195.

57. MOHP [Nepal]: Annual Report, Department of Health Services 2075/2076 (2018/2019). In.: Ministry of Health and Population. Kathmandu, Nepal. Ministry of Health and Population, Department of Health Services.; 2020.

58. Kruk ME, Gage AD, Arsenault C, Jordan K, Leslie HH, Roder-DeWan S, Adeyi $\mathrm{O}$, Barker P, Daelmans B, Doubova SV et al: High-quality health systems in the Sustainable Development Goals era: time for a revolution. Lancet Glob Health 2018, 6(11):e1196-e1252.

59. Munabi-Babigumira S, Glenton C, Lewin S, Fretheim A, Nabudere H: Factors that influence the provision of intrapartum and postnatal care by skilled birth attendants in low-and middle-income countries: a qualitative evidence synthesis. Cochrane Database Syst Rev 2017(11).

60. Bhatt H, Tiwari S, Ensor T, Ghimire DR, Gavidia T: Contribution of Nepal's free delivery care policies in improving utilisation of maternal health services. Int J Health Policy Manag 2018, 7(7):645-655. 


\section{Supplementary file}

2 Table 1 Description of variables included in the analysis of (dis)continuity of care of routine MNH services in Nepal, 2016

\begin{tabular}{|c|c|c|}
\hline Variables & Categories & Descriptions \\
\hline \multicolumn{3}{|l|}{ Independent } \\
\hline \multicolumn{3}{|l|}{ Structural } \\
\hline Wealth status & $\begin{array}{l}\text { Lower wealth status (lower } \\
40 \% \text { ); Upper wealth status } \\
\text { (upper } 60 \% \text { ) }\end{array}$ & $\begin{array}{l}\text { NDHS data had a variable wealth quintile, which was calculated based on scores } \\
\text { generated from principal components analysis of households' assets ( } 40 \text { items). It was } \\
\text { grouped into two categories: lower (poorest, poor, or collectively called as lower two } \\
\text { quintiles); and upper (middle, richer, and richest or collectively called as upper three } \\
\text { quintiles) wealth status. }\end{array}$ \\
\hline Ethnicity & Disadvantaged; Advantaged & $\begin{array}{l}\text { Disadvantaged: Dalit, Muslims, and Terai caste, Janajatis disadvantaged) and } \\
\text { advantaged: Brahmin/Chhetri, advantaged Janajatis). }\end{array}$ \\
\hline Maternal education & $\begin{array}{l}\text { Illiterate; Primary; Secondary } \\
\text { or higher }\end{array}$ & $\begin{array}{l}\text { Illiterate: Cannot read and write; Primary: } 1-8 \text { grade. } \\
\text { Secondary or higher: } 9^{\text {th }} \text { grade and higher }\end{array}$ \\
\hline Religion & Others; Hindu & Others include Muslims, Jain, Christian, Buddhist \\
\hline Maternal occupation & $\begin{array}{l}\text { Not working (housewife); } \\
\text { Agriculture; Working paid }\end{array}$ & $\begin{array}{l}\text { Based on the response of respondents, not working women are housewives (usually } \\
\text { husbands of such women have paid jobs), agriculture (family's main source of income is } \\
\text { agriculture), and paid job }\end{array}$ \\
\hline Perceived violence & No; Yes & $\begin{array}{l}\text { Yes: if women perceived beating in any one of the following conditions: the wife goes out } \\
\text { without telling the husband, wife neglects the children, wife argues with husband, wife } \\
\text { refuses to have sex with husband; wife burns the food. Otherwise no perceived violence }\end{array}$ \\
\hline Household head & Male; Female & This is the decision-maker in the family, as an indicator of women's empowerment \\
\hline Decision-making authority & No; Yes & $\begin{array}{l}\text { Whether women participated in at least one of the decisions regarding their health care, } \\
\text { purchases or visits to their family or relatives' households }\end{array}$ \\
\hline \multicolumn{3}{|r|}{ r } \\
\hline Languages & $\begin{array}{l}\text { Nepali, Maithili, Bhojpuri, and } \\
\text { Others (e.g. Tharu, Newari) }\end{array}$ & The primary language of the respondents \\
\hline Maternal age (in years) & $15-19,20-34,35$ and above & 15-19, and 35+ years are more at-risk groups \\
\hline Residence & Urban; Rural & $\begin{array}{l}\text { Municipalities are called urban, and remaining parts are called rural areas. This rural- } \\
\text { urbancategorizationis based on socioeconomic indicators of the population. Municipalities } \\
\text { have a higher populationand developmentindicators. However, many municipalities which } \\
\text { are considered as urban areas do not have adequate development facilities }\end{array}$ \\
\hline Province & 1-7 provinces & Now, provinces are numbered (has not been named) \\
\hline Region & Mountain; Hills; Terai & Ecological region \\
\hline Birth order & Less than $4 ; 4$ or more & Numbers of children in the family. \\
\hline
\end{tabular}




\begin{tabular}{|c|c|c|}
\hline Variables & Categories & Descriptions \\
\hline Sex of index child & Male; Female & Sex of last birth child \\
\hline Access to bank account & No; Yes & This is a marker of financial empowerment and access to financial resources \\
\hline Media exposure & No; Yes & $\begin{array}{l}\text { Received health related message from at least one of the following once a week: } \\
\text { newspaper, radio, or television }\end{array}$ \\
\hline Last birth (index child) & Unwanted; Wanted & Women perceived the youngest child intentional or not \\
\hline $\begin{array}{l}\text { Distance to health facilities is } \\
\text { a perceived problem }\end{array}$ & No problem; a big problem & Perception of problem associated with distance to HF for medical care \\
\hline \multicolumn{3}{|l|}{ Health system } \\
\hline $\begin{array}{l}\text { Perceived problem not } \\
\text { having female providers }\end{array}$ & No problem, big problem & Perceived problem, or not; if no female health provider for healthcare service delivery \\
\hline $\begin{array}{l}\text { Awareness on health } \\
\text { mothers' group }\end{array}$ & No; Yes & Awareness of health mothers' groups in the respective wards \\
\hline Mode of delivery & Normal; C-section & Types of childbirth services received by women at health facility \\
\hline \multicolumn{3}{|l|}{ Outcome variable } \\
\hline (Dis)continuity of care & $\begin{array}{l}\text { Discontinuation before } \\
\text { completing 4ANC visits, } \\
\text { discontinuation before } \\
\text { completing institutional } \\
\text { delivery, discontinuation of } \\
\text { before completing PNC visit; } \\
\text { completion of all three visits }\end{array}$ & (Dis)continuity of care from the antenatal- postnatal period \\
\hline
\end{tabular}

3 Table 2: Bivariable multinomial logistic regression analysis

\begin{tabular}{|c|c|c|c|c|}
\hline \multirow{2}{*}{ Determinants } & \multirow[t]{2}{*}{ Categories } & \multicolumn{3}{|c|}{ Unadjusted multinomial logistic regression analysis } \\
\hline & & $\begin{array}{l}\text { Completed <4ANC visits (vs all three } \\
\text { visits) Crude RR ( } 95 \% \mathrm{Cl} \text { ) }\end{array}$ & $\begin{array}{l}\text { Completed } \geq 4 A N C \text { visits (vs all three } \\
\text { visits) Crude RR }(95 \% \mathrm{Cl})\end{array}$ & $\begin{array}{l}\text { Completed } \geq 4 A N C \text { visits and institutional delivery } \\
\text { (vs all three visits) Crude RR }(95 \% \mathrm{Cl})\end{array}$ \\
\hline \multicolumn{5}{|r|}{ (90) } \\
\hline \multirow{2}{*}{ Wealth rank } & Upper & 1.00 & 1.00 & 1.00 \\
\hline & Lower $(60 \%)$ & $2.27(1.69,3.07)^{* * *}$ & $3.33(2.39,4.63)^{\star * *}$ & $0.97(0.66,1.42)$ \\
\hline \multirow[t]{2}{*}{ Ethnicity } & Advantaged & 1.00 & 1.00 & 1.00 \\
\hline & Disadvantaged & $3.01(2.08,4.35)^{* * *}$ & $1.96(1.40,2.73)^{\star * *}$ & $1.30(0.92,1.83)$ \\
\hline \multirow[t]{2}{*}{ Religion } & Other & 1.00 & 1.00 & 1.00 \\
\hline & Hindu & $0.58(0.38,0.89)^{*}$ & $0.96(0.61,1.51)$ & $1.00(0.59,1.68)$ \\
\hline \multirow[t]{3}{*}{ Maternal occupation } & Agriculture & 1.00 & 1.00 & 1.00 \\
\hline & Housewife & $1.10(0.83,1.45)$ & $0.50(0.37,0.68)^{\star \star \star *}$ & $1.19(0.80,1.76)$ \\
\hline & Working paid & $0.49(0.30,0.80)$ ** & $0.38(0.23,0.64)^{* \star *}$ & $1.02(0.58,1.79)$ \\
\hline
\end{tabular}




\begin{tabular}{|c|c|c|c|c|}
\hline \multirow{2}{*}{ Determinants } & \multirow{2}{*}{ Categories } & \multicolumn{3}{|c|}{ Unadjusted multinomial logistic regression analysis } \\
\hline & & $\begin{array}{l}\text { Completed <4ANC visits (vs all three } \\
\text { visits) Crude RR }(95 \% \mathrm{Cl})\end{array}$ & $\begin{array}{l}\text { Completed } \geq 4 A N C \text { visits (vs all three } \\
\text { visits) Crude RR }(95 \% \mathrm{Cl})\end{array}$ & $\begin{array}{l}\text { Completed } \geq 4 A N C \text { visits and institutional delivery } \\
\text { (vs all three visits) Crude } R R(95 \% \mathrm{Cl})\end{array}$ \\
\hline \multirow{3}{*}{ Maternal education } & Higher & 1.00 & 1.00 & 1.00 \\
\hline & Illiterate & $6.16(4.38,8.65)^{\star \star \star}$ & $3.00(2.11,4.25)^{\star \star \star}$ & $1.34(0.86,2.08)$ \\
\hline & Primary & $4.15(2.94,5.87)^{* \star *}$ & $3.18(2.17,4.67)^{* * *}$ & $1.32(0.83,2.08)$ \\
\hline \multirow[t]{2}{*}{ Perceived violence } & No & 1.00 & 1.00 & 1.00 \\
\hline & Yes & $1.43(1.07,1.91)^{*}$ & $1.28(0.92,1.79)$ & $1.51(1.06,2.15)^{*}$ \\
\hline \multirow[t]{2}{*}{ Decision making } & Yes & 1.00 & 1.00 & 1.00 \\
\hline & No & $1.39(1.06,1.83)^{*}$ & $1.26(0.89,1.80)$ & $1.06(0.76,1.49)$ \\
\hline \multirow[t]{2}{*}{ Household head } & Male & 1.00 & 1.00 & 1.00 \\
\hline & Female & $0.72(0.55,0.93)^{*}$ & $0.76(0.55,1.04)$ & $0.77(0.52,1.15)$ \\
\hline \multicolumn{5}{|l|}{ Intermediary } \\
\hline \multirow[t]{4}{*}{ Language } & Nepali & 1.00 & 1.00 & 1.00 \\
\hline & Maithili & $2.52(1.61,3.96)^{\star * \star}$ & $2.11(1.29,3.43)$ ** & $1.30(0.81,2.08)$ \\
\hline & Bhojpuri & $6.36(3.84,10.55)^{\star \star \star}$ & $1.57(0.81,3.05)$ & $2.13(1.06,4.26)^{*}$ \\
\hline & Others & $1.70(1.16,2.49)^{* *}$ & $1.18(0.80,1.72)$ & $0.89(0.60,1.31)$ \\
\hline \multirow[t]{7}{*}{ Province } & One & 1.00 & 1.00 & 1.00 \\
\hline & Two & $3.50(2.09,5.84)^{* * *}$ & $1.47(0.84,2.59)$ & $2.56(1.32,4.96)^{\star *}$ \\
\hline & Three & $1.07(0.56,2.02)$ & $0.58(0.29,1.15)$ & $1.20(0.61,2.36)$ \\
\hline & Four & $1.02(0.57,1.81)$ & $0.45(0.22,0.90)$ * & $0.95(0.46,1.96)$ \\
\hline & Five & $1.36(0.73,2.52)$ & $0.97(0.59,1.60)$ & $1.86(0.97,3.58)$ \\
\hline & Six & $4.30(2.55,7.25)^{* * *}$ & $1.93(1.04,3.58)$ * & $2.19(1.07,4.47)^{*}$ \\
\hline & Seven & $0.79(0.47,1.32)$ & $0.70(0.37,1.33)$ & $2.89(1.51,5.54)^{* *}$ \\
\hline \multirow{3}{*}{ Region } & Hill & 1.00 & 1.00 & 1.00 \\
\hline & Mountain & $1.46(0.72,2.97)$ & $2.05(1.04,4.02)$ * & $0.35(0.11,1.10)$ \\
\hline & Terai & $1.74(1.22,2.48)^{* *}$ & $1.17(0.82,1.66)$ & $1.40(0.98,2.00)$ \\
\hline \multirow[t]{2}{*}{ Residence } & Urban & 1.00 & 1.00 & 1.00 \\
\hline & Rural & $2.12(1.48,3.04)^{\star \star *}$ & $2.51(1.76,3.59)^{\star \star *}$ & $1.12(0.77,1.61)$ \\
\hline \multirow[t]{3}{*}{ Maternal age (in years) } & $15-19$ & 1.00 & 1.00 & 1.00 \\
\hline & $20-34$ & $1.15(0.76,1.74)$ & $1.22(0.80,1.86)$ & $0.67(0.44,1.01)$ \\
\hline & 35 above & $1.88(0.96,3.66)$ & $1.62(0.69,3.80)$ & $0.78(0.32,1.91)$ \\
\hline \multirow[t]{2}{*}{ Birth order } & $<4$ & 1.00 & 1.00 & 1.00 \\
\hline & $\geq 4$ & $4.54(3.09,6.68)^{* \star *}$ & $2.70(1.88,3.89)^{\star \star \star}$ & $0.71(0.38,1.34)$ \\
\hline \multirow[t]{2}{*}{ Sex of index child } & Male & 1.00 & 1.00 & 1.00 \\
\hline & Female & $1.08(0.85,1.37)$ & $1.18(0.88,1.58)$ & $1.02(0.75,1.38)$ \\
\hline \multirow[t]{2}{*}{ Access to bank account } & Yes & 1.00 & 1.00 & 1.00 \\
\hline & No & $2.89(2.02,4.14)^{* * \star}$ & $2.23(1.62,3.07)^{\star \star \star}$ & $1.44(1.01,2.06)^{*}$ \\
\hline \multirow[t]{2}{*}{ Media exposure } & Yes & 1.00 & 1.00 & 1.00 \\
\hline & No & $3.72(2.85,4.85)^{\star * \star}$ & $2.83(2.12,3.79)^{\star \star \star}$ & $1.27(0.90,1.78)$ \\
\hline \multirow[t]{2}{*}{ Last birth (index child) } & Wanted & & & \\
\hline & Unwanted & $1.95(1.38,2.76)^{\star * \star}$ & $1.12(0.76,1.66)$ & $1.18(0.75,1.87)$ \\
\hline \multirow[t]{2}{*}{$\begin{array}{l}\text { Distance to health } \\
\text { facilities was a } \\
\text { perceived problem }\end{array}$} & No problem & 1.00 & 1.00 & 1.00 \\
\hline & Big problem & $2.17(1.64,2.89)^{\star * \star}$ & $2.39(1.75,3.25)^{\star * \star}$ & $1.47(1.03,2.08)^{*}$ \\
\hline
\end{tabular}




\begin{tabular}{|c|c|c|c|c|}
\hline \multirow[t]{2}{*}{ Determinants } & \multirow[t]{2}{*}{ Categories } & \multicolumn{3}{|c|}{ Unadjusted multinomial logistic regression analysis } \\
\hline & & $\begin{array}{l}\text { Completed <4ANC visits (vs all three } \\
\text { visits) Crude RR ( } 95 \% \mathrm{Cl})\end{array}$ & $\begin{array}{l}\text { Completed } \geq 4 \text { ANC visits (vs all three } \\
\text { visits) Crude RR ( } 95 \% \mathrm{Cl})\end{array}$ & $\begin{array}{l}\text { Completed } \geq 4 A N C \text { visits and institutional delivery } \\
\text { (vs all three visits) Crude RR ( } 95 \% \mathrm{Cl})\end{array}$ \\
\hline \multicolumn{5}{|l|}{ Health system } \\
\hline \multirow[t]{2}{*}{$\begin{array}{l}\text { Perceived problem not } \\
\text { having female providers }\end{array}$} & No problem & 1.00 & 1.00 & 1.00 \\
\hline & Big problem & $2.16(1.56,2.99)^{* * *}$ & $2.13(1.50,3.02)^{* * *}$ & $1.74(1.23,2.46)^{\star *}$ \\
\hline \multirow[t]{2}{*}{$\begin{array}{l}\text { Awareness on health } \\
\text { mothers' group }\end{array}$} & Yes & 1.00 & 1.00 & 1.00 \\
\hline & No & $1.75(1.36,2.25)^{\star \star \star}$ & $1.06(0.79,1.41)$ & $1.07(0.77,1.48)$ \\
\hline \multirow[t]{2}{*}{ Mode of delivery } & C-section & 1.00 & & 1.00 \\
\hline & Normal & $3.62(2.04,6.45)^{\star \star \star}$ & & $1.73(1.06,2.84)$ * \\
\hline
\end{tabular}


